\title{
NLO QCD procedure of the semi-inclusive deep inelastic scattering data analysis with respect to the light quark polarized sea
}

\author{
A.N. Sissakian ${ }^{1}$, O.Yu. Shevchenko ${ }^{2}$, O.N. Ivanov ${ }^{3}$
}

Joint Institute for Nuclear Research

\begin{abstract}
The semi-inclusive deep inelastic scattering (SIDIS) process is considered. A theoretical procedure is proposed allowing the direct extraction from the SIDIS data of the first moments of the polarized valence distributions and of the first moment difference of the light sea quark polarized distributions in the next to leading QCD order. The validity of the procedure is confirmed by the respective simulations

PACS numbers: 13.85.Ni, 13.60.Hb, 13.88.+e
\end{abstract}

\section{Introduction}

The extraction of the polarized quark and gluon densities is one of the main tasks of the semiinclusive deep inelastic scattering (SIDIS) experiments with the polarized beam and target. Of a special importance for the modern SIDIS experiments are the questions of strange quark and gluon contributions to the nucleon spin, and, also the sea quark share as well as the possibility of broken sea scenario. Indeed, it is known [1] that the unpolarized sea of light quarks is asymmetric, so that the first moments of the unpolarized $\bar{u}$ and $\bar{d}$ quark densities do not equal to each other:

$$
\int_{0}^{1} d x[\bar{d}(x)-\bar{u}(x)]=0.147 \pm 0.039 \neq 0 .
$$

The question arises: does the analogous situation occurs in the polarized case, i.e. whether the polarized density $\Delta \bar{u}$ and its first moment ${ }^{4} \Delta_{1} \bar{u} \equiv \int_{0}^{1} d x \Delta \bar{u}$ are respectively equal to $\Delta \bar{d}$ and $\Delta_{1} \bar{d}$ or not.

In ref. 2] the possibility of broken sea scenario was analyzed, considering the results of SIDIS experiments on $\Delta q$ with respect to their consistence with the Bjorken sum rule (BSR) predictions. It was shown [2] that using the results of [3] on the valence quark distributions $\Delta_{1} q_{V}$ obtained in the leading (LO) QCD order, one can immediately estimate the first moment difference of the $u$ and $d$ sea quark polarized distributions:

$$
\Delta_{1} \bar{u}-\Delta_{1} \bar{d}=0.235 \pm 0.097 .
$$

\footnotetext{
${ }^{1}$ E-mail address: sisakian@jinr.ru

${ }^{2}$ E-mail address: shevch@nusun.jinr.ru

${ }^{3}$ E-mail address: ivon@jinr.ru

${ }^{4}$ From now on the notation $\Delta_{1} q \equiv \int_{0}^{1} d x \Delta q$ will be used to distinguish the local in Bjorken $x$ polarized quark densities $\Delta q(x)$ and their first moments.
} 
At the same time, it was stressed in [2] that this is just a speculation, and, to get the reliable results on $\Delta q$ from the data obtained at the relatively small average $Q^{2}=2.5 \mathrm{GeV}^{2}$ [3], one should apply NLO QCD analysis. The main goal of this paper is to present such a NLO QCD procedure allowing the direct extraction of the quantity $\Delta_{1} \bar{u}-\Delta_{1} \bar{d}$ from the SIDIS data.

It is known that the description of semi-inclusive DIS processes turns out to be much more complicated in comparison with the inclusive polarized DIS. First, the fragmentation functions are involved, for which the information is limited ${ }^{5}$. Second, the extraction of the quark densities in NLO QCD order turns out to be rather difficult, since the double convolution products are involved. So, to achieve a reliable description of the SIDIS data it is very desirable, on the one hand, to exclude from consideration the fragmentation functions, whenever possible, and, on the other hand, to try to simplify the NLO considerations as much as possible.

It is well known (see, for example, 4] and references therein) that within LO QCD approximation one can completely exclude the fragmentation functions from the expressions for the valence quark polarized distributions $\Delta q_{V}$ through experimentally measured asymmetries. To this end, instead of the usual virtual photon asymmetry $A_{\gamma N}^{h} \equiv A_{1 N}^{h}$ (which is expressed in terms of the directly measured asymmetry $A_{\text {exp }}^{h}=\left(n_{\uparrow \downarrow}^{h}-n_{\uparrow \uparrow}^{h}\right) /\left(n_{\uparrow \downarrow}^{h}+n_{\uparrow \uparrow}^{h}\right)$ as $\left.A_{1 N}^{h}=\left(P_{B} P_{T} f D\right)^{-1} A_{\text {exp }}^{h}\right)$, one has to measure so called "difference asymmetry" $A_{N}^{h-\bar{h}}$ which is expressed in terms of the respective counting rates ${ }^{6}$ as

$$
A_{N}^{h-\bar{h}}\left(x, Q^{2} ; z\right)=\frac{1}{P_{B} P_{T} f D} \frac{\left(n_{\uparrow \downarrow}^{h}-n_{\uparrow \downarrow}^{\bar{h}}\right)-\left(n_{\uparrow \uparrow}^{h}-n_{\uparrow \uparrow}^{\bar{h}}\right)}{\left(n_{\uparrow \downarrow}^{h}-n_{\uparrow \downarrow}^{\bar{h}}\right)+\left(n_{\uparrow \uparrow}^{h}-n_{\uparrow \uparrow}^{\bar{h}}\right)},
$$

where the event densities $n_{\uparrow \downarrow(\uparrow \uparrow)}^{h}=d N_{\uparrow \downarrow(\uparrow \uparrow)}^{h} / d z$, i.e. $n_{\uparrow \downarrow(\uparrow \uparrow)}^{h} d z$ are the numbers of events for anti-parallel (parallel) orientations of incoming lepton and target nucleon spins for the hadrons of type $\mathrm{h}$ registered in the interval $d z$. Quantities $P_{B}$ and $P_{T}, \mathrm{f}$ and $\mathrm{D}$ are the beam and target polarizations, dilution and depolarization factors, respectively (for details on these quantities see, for example, [5, 6] and references therein). Then, the LO theoretical expressions for the difference asymmetries look like (see, for example, COMPASS proposal [7], appendix A)

$$
\begin{array}{ll}
A_{p}^{\pi^{+}-\pi^{-}}=\frac{4 \Delta u_{V}-\Delta d_{V}}{4 u_{V}-d_{V}} ; & A_{d}^{\pi^{+}-\pi^{-}}=\frac{\Delta u_{V}+\Delta d_{V}}{u_{V}+d_{V}} ; \\
A_{n}^{\pi^{+}-\pi^{-}}=\frac{4 \Delta d_{V}-\Delta u_{V}}{4 d_{V}-u_{V}} ; & A_{p}^{K^{+}-K^{-}}=\frac{\Delta u_{V}}{u_{V}} ; \quad A_{d}^{K^{+}-K^{-}}=A_{d}^{\pi^{+}-\pi^{-}},
\end{array}
$$

i.e., on the one hand, they contain only valence quark polarized densities, and, on the other hand, have the remarkable property to be free of any fragmentation functions.

\section{Theoretical basis of the procedure}

Let us start NLO consideration with the known [4, 8, 9, 10, theoretical expressions for the difference asymmetries

$$
A_{N}^{h-\bar{h}}\left(x, Q^{2} ; z\right)=\frac{g_{1}^{N / h}-g_{1}^{N / \bar{h}}}{\tilde{F}_{1}^{N / h}-\tilde{F}_{1}^{N / \bar{h}}} \quad(N=p, n, d),
$$

\footnotetext{
${ }^{5}$ For discussion of this subject see, for example [4] and references therein.

6 As usual, one should realize the quantities $n_{\uparrow \uparrow(\uparrow \downarrow)}^{h}$ entering Eq. (2) not as the pure event densities but as the event densities multiplied by the respective luminosities which, in general, do not cancel out - see the Appendix.
} 
where the semi-inclusive analogs of the structure functions $g_{1}^{N}$ and $F_{1}^{N}$, functions $g_{1}^{N / h}$ and $\tilde{F}_{1}^{N / h}$, are related to the respective polarized and unpolarized semi-inclusive differential cross-sections as follows 9 ]

$$
\begin{aligned}
\frac{d^{3} \sigma_{N \uparrow \downarrow}^{h}}{d x d y d z}-\frac{d^{3} \sigma_{N \uparrow \uparrow}^{h}}{d x d y d z} & =\frac{4 \pi \alpha^{2}}{Q^{2}}(2-y) g_{1}^{N / h}\left(x, z, Q^{2}\right) \\
\frac{d^{3} \sigma_{N}^{h}}{d x d y d z} & =\frac{2 \pi \alpha^{2}}{Q^{2}} \frac{1+(1-y)^{2}}{y} 2 \tilde{F}_{1}^{N / h}\left(x, z, Q^{2}\right)
\end{aligned}
$$

The semi-inclusive structure functions $g_{1}^{p(n) / h}$ are given in NLO by

$$
\begin{aligned}
& g_{1}^{p / h}= \sum_{q, \bar{q}} e_{q}^{2} \Delta q\left[1+\otimes \frac{\alpha_{s}}{2 \pi} \delta C_{q q} \otimes\right] D_{q}^{h} \\
&+\left(\sum_{q, \bar{q}} e_{q}^{2} \Delta q\right) \otimes \frac{\alpha_{s}}{2 \pi} \delta C_{g q} \otimes D_{g}^{h} \\
&+ \Delta g \otimes \frac{\alpha_{s}}{2 \pi} \delta C_{q g} \otimes\left(\sum_{q, \bar{q}} e_{q}^{2} D_{q}^{h}\right), \\
& g_{1}^{n / h}=\left.g_{1}^{p / h}\right|_{u \leftrightarrow d}
\end{aligned}
$$

where the double convolution product is defined as

$$
[\Delta q \otimes \delta C \otimes D](x, z) \equiv \int_{\mathcal{D}} \int \frac{d x^{\prime}}{x^{\prime}} \frac{d z^{\prime}}{z^{\prime}} \Delta q\left(\frac{x}{x^{\prime}}\right) \delta C\left(x^{\prime}, z^{\prime}\right) D\left(\frac{z}{z^{\prime}}\right) .
$$

The respective expressions for $2 \tilde{F}_{1}^{p(n) / h}$ have the form analogous to Eq. (17) with the substitution $\Delta q \rightarrow q, \delta C \rightarrow \tilde{C}$. The expressions for the Wilson coefficients $\delta C_{q q(q g, g q)}$ and $\tilde{C}_{q q(q g, g q)} \equiv C_{q q(q g, g q)}^{1}+2(1-y) /\left(1+(1-y)^{2}\right) C_{q q(q g, g q)}^{L}$ can be found, for example, in [9], Appendix $\mathrm{C}$.

It is remarkable that due to the properties of the fragmentation functions:

$$
\begin{aligned}
& D_{1} \equiv D_{u}^{\pi^{+}}=D_{\bar{u}}^{\pi^{-}}=D_{\bar{d}}^{\pi^{+}}=D_{d}^{\pi^{-}}, \\
& D_{2} \equiv D_{d}^{\pi^{+}}=D_{\bar{d}}^{\pi^{-}}=D_{u}^{\pi^{-}}=D_{\bar{u}}^{\pi^{+}},
\end{aligned}
$$

in the differences $g_{1}^{p / \pi^{+}}-g_{1}^{p / \pi^{-}}$and $\tilde{F}_{1}^{p / \pi^{+}}-\tilde{F}_{1}^{p / \pi^{-}}$(and, therefore, in the asymmetries $A_{p}^{\pi^{+}-\pi^{-}}$ and $A_{d}^{\pi^{+}-\pi^{-}}$) only the contributions containing the Wilson coefficients $\delta C_{q q}$ and $\tilde{C}_{q q}$ survive. However, even then the system of double integral equations

$$
\begin{aligned}
& A_{p}^{\pi^{+}-\pi^{-}}\left(x, Q^{2} ; z\right)=\frac{\left(4 \Delta u_{V}-\Delta d_{V}\right)\left[1+\otimes \alpha_{s} /(2 \pi) \delta C_{q q} \otimes\right]\left(D_{1}-D_{2}\right)}{\left(4 u_{V}-d_{V}\right)\left[1+\otimes \alpha_{s} /(2 \pi) C_{q q} \otimes\right]\left(D_{1}-D_{2}\right)}, \\
& A_{n}^{\pi^{+}-\pi^{-}}\left(x, Q^{2} ; z\right)=\left.A_{p}^{\pi^{+}-\pi^{-}}\left(x, Q^{2} ; z\right)\right|_{u_{V} \leftrightarrow d_{V}}
\end{aligned}
$$

proposed by E. Christova and E. Leader [4, is rather difficult to solve directly ${ }^{7}$ with respect to the local quantities $\Delta u_{V}\left(x, Q^{2}\right)$ and $\Delta d_{V}\left(x, Q^{2}\right)$. Besides, the range of integration $\mathcal{D}$ used in

${ }^{7}$ So that it seems that the only real possibility to extract polarized distributions from the data in NLO QCD order is to use some proper fit. At the same time, it is known that a such procedure is rather ambiguous since the sea distributions are very sensitive to the choice of initial functions for $\Delta q$ parametrization (and, especially, for $\Delta \bar{q}$ parametrization). 
4] has a very complicated form, namely:

$$
\frac{x}{x+(1-x) z} \leq x^{\prime} \leq 1 \text { with } z \leq z^{\prime} \leq 1
$$

if $x+(1-x) z \geq 1$, and, additionally, range

$$
x \leq x^{\prime} \leq x /(x+(1-x)) z
$$

with $x\left(1-x^{\prime}\right) /\left(x^{\prime}(1-x)\right) \leq z^{\prime} \leq 1$ if $x+(1-x) z \leq 1$. Such enormous complication of the convolution integral range occurs if one introduces (to take into account the target fragmentation contributions $^{8}$ and to exclude the cross-section singularity problem at $z_{h}=0$ ) a new hadron kinematic variable $z=E_{h} / E_{N}(1-x)(\gamma p$ c.m. frame) instead of the usual semi-inclusive variable $z_{h}=(P h) /(P q)=$ (labsystem) $E_{h} / E_{\gamma}$. However, both problems compelling us to introduce $z$, instead of $z_{h}$, can be avoided (see, for example [9, 10]) if one, just to neglect the target fragmentation, applies a proper kinematical cut $Z<z_{h} \leq 1$, i.e. properly restricts the kinematical region covered by the final state hadrons ${ }^{9}$. Then, one can safely use, instead of $\mathrm{z}$, the usual variable $z_{h}$, which at once makes the integration range $\mathcal{D}$ in the double convolution product (9) very simple: $x \leq x^{\prime} \leq 1, z_{h} \leq z^{\prime} \leq 1$. Note that in applying the kinematical cut it is much more convenient to deal with the total numbers of events (multiplied by the respective luminosities - see footnote 6 and the Appendix)

$$
\left.N_{\uparrow \downarrow(\uparrow \uparrow)}^{h}\left(x, Q^{2}\right)\right|_{Z}=\int_{Z}^{1} d z_{h} n_{\uparrow \downarrow(\uparrow \uparrow)}^{h}\left(x, Q^{2} ; z_{h}\right)
$$

within the entire interval $Z \leq z_{h} \leq 1$ and the respective integral difference asymmetries ${ }^{10}$

$$
\begin{aligned}
\left.A_{N}^{h-\bar{h}}\left(x, Q^{2}\right)\right|_{Z} & =\left.\frac{1}{P_{B} P_{T} f D} \frac{\left(N_{\uparrow \downarrow}^{h}-N_{\uparrow \downarrow}^{\bar{h}}\right)-\left(N_{\uparrow \uparrow}^{h}-N_{\uparrow \uparrow}^{\bar{h}}\right)}{\left(N_{\uparrow \downarrow}^{h}-N_{\uparrow \downarrow}^{\bar{h}}\right)+\left(N_{\uparrow \uparrow}^{h}-N_{\uparrow \uparrow}^{\bar{h}}\right)}\right|_{Z}= \\
& =\frac{\int_{Z}^{1} d z_{h}\left(g_{1}^{N / h}-g_{1}^{N / \bar{h}}\right)}{\int_{Z}^{1} d z_{h}\left(\tilde{F}_{1}^{N / h}-\tilde{F}_{1}^{N / \bar{h}}\right)} \quad(N=p, n, d),
\end{aligned}
$$

than with the local in $z_{h}$ quantities $n_{\uparrow \downarrow(\uparrow \uparrow)}\left(x, Q^{2} ; z_{h}\right)$ and $A_{N}^{h-\bar{h}}\left(x, Q^{2} ; z_{h}\right)$. So, the expressions for the proton and deutron integral difference asymmetries assume the form ${ }^{11}$

$$
\begin{gathered}
\left.A_{p}^{\pi^{+}-\pi^{-}}\left(x, Q^{2}\right)\right|_{Z}=\frac{\left(4 \Delta u_{V}-\Delta d_{V}\right) \int_{Z}^{1} d z_{h}\left[1+\otimes \frac{\alpha_{s}}{2 \pi} \delta C_{q q} \otimes\right]\left(D_{1}-D_{2}\right)}{\left(4 u_{V}-d_{V}\right) \int_{Z}^{1} d z_{h}\left[1+\otimes \frac{\alpha_{s}}{2 \pi} \tilde{C}_{q q} \otimes\right]\left(D_{1}-D_{2}\right)}, \\
\left.A_{d}^{\pi^{+}-\pi^{-}}\left(x, Q^{2}\right)\right|_{Z}=\frac{\left(\Delta u_{V}+\Delta d_{V}\right) \int_{Z}^{1} d z_{h}\left[1+\otimes \frac{\alpha_{s}}{2 \pi} \delta C_{q q} \otimes\right]\left(D_{1}-D_{2}\right)}{\left(u_{V}+d_{V}\right) \int_{Z}^{1} d z_{h}\left[1+\otimes \frac{\alpha_{s}}{2 \pi} \tilde{C}_{q q} \otimes\right]\left(D_{1}-D_{2}\right)},
\end{gathered}
$$

\footnotetext{
${ }^{8}$ Then, one should also add the target fragmentation contributions to the right-hand side of Eq. (17).

${ }^{9}$ This is just what was done in the HERMES and SMC experiments, where the applied kinematical cut was $z_{h}>Z=0.2$.

${ }^{10}$ Namely the integral spin symmetries $A_{1 N}^{h}=\int_{Z}^{1} d z_{h} g_{1 N}^{h} / \int_{Z}^{1} d z_{h} \tilde{F}_{1 N}^{h}$ were measured by SMC and HERMES experiments (see [3, 5, 6] and also [10]).

${ }^{11}$ Here one uses the equality $g_{1}^{d / h} \simeq g_{1}^{p / h}+g_{1}^{n / h}$ which is valid up to corrections of order $O\left(\omega_{D}\right)$, where $\omega_{D}=0.05 \pm 0.01$ is the probability to find deutron in the $D$-state.
} 
where the double convolution product reads

$$
[\Delta q \otimes \delta C \otimes D]\left(x, z_{h}\right)=\int_{x}^{1} \frac{d x^{\prime}}{x^{\prime}} \int_{z_{h}}^{1} \frac{d z^{\prime}}{z^{\prime}} \Delta q\left(\frac{x}{x^{\prime}}\right) \delta C\left(x^{\prime}, z^{\prime}\right) D\left(\frac{z_{h}}{z^{\prime}}\right) .
$$

With a such simple convolution region, one can apply the well known property of the n-th Melin moments $M^{n}(f) \equiv \int_{0}^{1} d x x^{n-1} f(x)$ to split the convolution product into a simple product of the Melin moments of the respective functions:

$$
M^{n}[A \otimes B] \equiv \int_{0}^{1} d x x^{n-1} \int_{x}^{1} \frac{d y}{y} A\left(\frac{x}{y}\right) B(y)=M^{n}(A) M^{n}(B) .
$$

So, applying the first moment to the difference asymmetries $\left.A_{p}^{\pi^{+}-\pi^{-}}\left(x, Q^{2}\right)\right|_{Z}$ and $\left.A_{d}^{\pi^{+}-\pi^{-}}\left(x, Q^{2}\right)\right|_{Z}$, given by (14), (15), one gets a system of two equations for $\Delta_{1} u_{V} \equiv \int_{0}^{1} d x \Delta u_{V}$ and $\Delta_{1} d_{V} \equiv$ $\int_{0}^{1} d x \Delta d_{V}$ :

$$
\begin{aligned}
& \left(4 \Delta_{1} u_{V}-\Delta_{1} d_{V}\right)\left(L_{1}-L_{2}\right)=\mathcal{A}_{p}^{\exp } \\
& \left(\Delta_{1} u_{V}+\Delta_{1} d_{V}\right)\left(L_{1}-L_{2}\right)=\mathcal{A}_{d}^{\exp }
\end{aligned}
$$

with the solution

$$
\Delta_{1} u_{V}=\frac{1}{5} \frac{\mathcal{A}_{p}^{\text {exp }}+\mathcal{A}_{d}^{\text {exp }}}{L_{1}-L_{2}} ; \quad \Delta_{1} d_{V}=\frac{1}{5} \frac{4 \mathcal{A}_{d}^{\text {exp }}-\mathcal{A}_{p}^{\text {exp }}}{L_{1}-L_{2}}
$$

Here we introduce the notation

$$
\begin{gathered}
\left.\mathcal{A}_{p}^{\exp } \equiv \int_{0}^{1} d x A_{p}^{\pi^{+}-\pi^{-}}\right|_{Z}\left(4 u_{V}-d_{V}\right) \int_{Z}^{1} d z_{h}\left[1+\otimes \frac{\alpha_{s}}{2 \pi} \tilde{C}_{q q} \otimes\right]\left(D_{1}-D_{2}\right), \\
\left.\mathcal{A}_{d}^{\text {exp }} \equiv \int_{0}^{1} d x A_{d}^{\pi^{+}-\pi^{-}}\right|_{Z}\left(u_{V}+d_{V}\right) \int_{Z}^{1} d z_{h}\left[1+\otimes \frac{\alpha_{s}}{2 \pi} \tilde{C}_{q q} \otimes\right]\left(D_{1}-D_{2}\right) \\
L_{1} \equiv L_{u}^{\pi^{+}}=L_{\bar{u}}^{\pi^{-}}=L_{\bar{d}}^{\pi^{+}}=L_{d}^{\pi^{-}}, \\
L_{2} \equiv L_{d}^{\pi^{+}}=L_{\bar{d}}^{\pi^{-}}=L_{u}^{\pi^{-}}=L_{\bar{u}}^{\pi^{+}},
\end{gathered}
$$

where

$$
L_{q}^{h} \equiv \int_{Z}^{1} d z_{h}\left[D_{q}^{h}\left(z_{h}\right)+\frac{\alpha_{s}}{2 \pi} \int_{z_{h}}^{1} \frac{d z^{\prime}}{z^{\prime}} \Delta_{1} C\left(z^{\prime}\right) D_{q}^{h}\left(\frac{z_{h}}{z^{\prime}}\right)\right]
$$

with the coefficient $\Delta_{1} C(z) \equiv \int_{0}^{1} d x \delta C_{q q}(x, z)$.

Now one may do the last step to get the NLO QCD equation for the extraction of the quantity $\Delta_{1} \bar{u}-\Delta_{1} \bar{d}$ we are interesting in. Namely, on can use the equivalent of BSR (see [2] and references therein for details) rewritten in terms of the valence and sea distributions:

$$
\Delta_{1} \bar{u}-\Delta_{1} \bar{d}=\frac{1}{2}\left|\frac{g_{A}}{g_{V}}\right|-\frac{1}{2}\left(\Delta_{1} u_{V}-\Delta_{1} d_{V}\right) .
$$


Using Eqs. (20, 24) one gets a simple expression for the quantity $\Delta_{1} \bar{u}-\Delta_{1} \bar{d} \equiv \int_{0}^{1} d x\left(\Delta \bar{u}\left(x, Q^{2}\right)-\right.$ $\left.\Delta \bar{d}\left(x, Q^{2}\right)\right)$ in terms of experimentally measured quantities, that is valid in NLO QCD :

$$
\Delta_{1} \bar{u}-\Delta_{1} \bar{d}=\frac{1}{2}\left|\frac{g_{A}}{g_{V}}\right|-\frac{2 \mathcal{A}_{p}^{e x p}-3 \mathcal{A}_{d}^{e x p}}{10\left(L_{1}-L_{2}\right)} .
$$

It is easy to see that all the quantities present in the right-hand side of (25), with the exception of the two difference asymmetries $\left.A_{p}^{\pi^{+}-\pi^{-}}\right|_{Z}$ and $\left.A_{d}^{\pi^{+}-\pi^{-}}\right|_{Z}\left(\right.$ entering $\mathcal{A}_{p}^{\text {exp }}$ and $\mathcal{A}_{d}^{\text {exp }}$, respectively) can be extracted from unpolarized ${ }^{12}$ semi - inclusive data and can, thus, be considered here as a known input. So, the only quantities that have to be measured in polarized semi-inclusive DIS are the difference asymmetries $\left.A_{p}^{\pi^{+}-\pi^{-}}\right|_{Z}$ and $\left.A_{d}^{\pi^{+}-\pi^{-}}\right|_{Z}$ which, in turn, are just simple combinations of the directly measured counting rates.

\section{Errors on the difference asymmetries}

To check the validity of the proposed procedure let us perform the respective simulations. To this end one can use the polarized event generator PEPSI [13]. First of all, let us clarify the very important issue of the errors on the difference asymmetries. At first sight it could seem that the difference asymmetries suffer from the much larger errors in comparison with the usual asymmetries. Indeed, the approximate formula for the estimation of the statistical error on the difference asymmetries reads (see Eqs. (A.15), (A.16) in the Appendix)

$$
\delta\left(A_{p(n, d)}^{\pi^{+}-\pi^{-}}\right) \sim \frac{\sqrt{N^{\pi^{+}}+N^{\pi^{-}}}}{N^{\pi^{+}}-N^{\pi^{-}}} .
$$

So, one can see that, contrary to the usual asymmetries, the difference of the total (for both parallel and anti-parallel beam and target polarizations) counting rates for $\pi^{+}$and $\pi^{-}$ production, $N^{\pi^{+}} \equiv N_{\uparrow \downarrow}^{\pi^{+}}+N_{\uparrow \uparrow}^{\pi^{+}}$and $N^{\pi^{-}} \equiv N_{\uparrow \downarrow}^{\pi^{-}}+N_{\uparrow \uparrow}^{\pi^{-}}$, occurs in the denominator of the expression for $\delta A_{p(n, d)}^{\pi^{+}-\pi^{-}}$, and it could lead to the large statistical errors on this asymmetry. Such situation indeed occurs for the neutron target. However, fortunately, for the proton and deutron targets there is an important circumstance which rescues the situation.

The point is that, unlike the neutron target case (see Fig. 1), the production of positive pions on the proton target (see Fig. 2), in the widest region in Bjorken $x^{13}$, essentially exceeds the production of negative pions, whereas for the deuteron target the difference in $\pi^{+}$and $\pi^{-}$ production is not so drastic but is still essential (see Fig. 3).

It is of importance that though the histograms in Figs. $1-3$ are obtained using the PEPSI event generator (just summing the events with parallel and anti-parallel beam and target polarizations), they represent the general, well established experimentally [14 picture ( see section 4.3 and Fig. 10 in ref. [14]), and peculiar to all known SIDIS event generators ${ }^{14}$.

\footnotetext{
${ }^{12}$ The spin-independent fragmentation functions D can be taken either from independent measurements of $e^{+} e^{-}$- annihilation into hadrons [1] or from the hadron production in unpolarized DIS [12].

13 This occurs everywhere except for the vicinity of $x_{B}=0$, where the ratio $N^{\pi^{+}} / N^{\pi^{-}}$approaches unity owing to the dominant contribution of the sea quarks [14. However, let us stress that for the statistical error only the difference $N^{\pi^{+}}-N^{\pi-}$ is of importance, and, at the statistics available to HERMES and COMPASS, $N^{\pi^{+}}-N^{\pi-}$ is not a small quantity even in the vicinity of the minimal value $x_{B}=0.003$ accessible to measurement - see below.

${ }^{14}$ Absolutely the same histograms for $N_{n, p, d}^{\pi^{+}}$and $N_{n, p, d}^{\pi^{-}}$are reproduced using, for example, the unpolarized event generator LEPTO [15].
} 


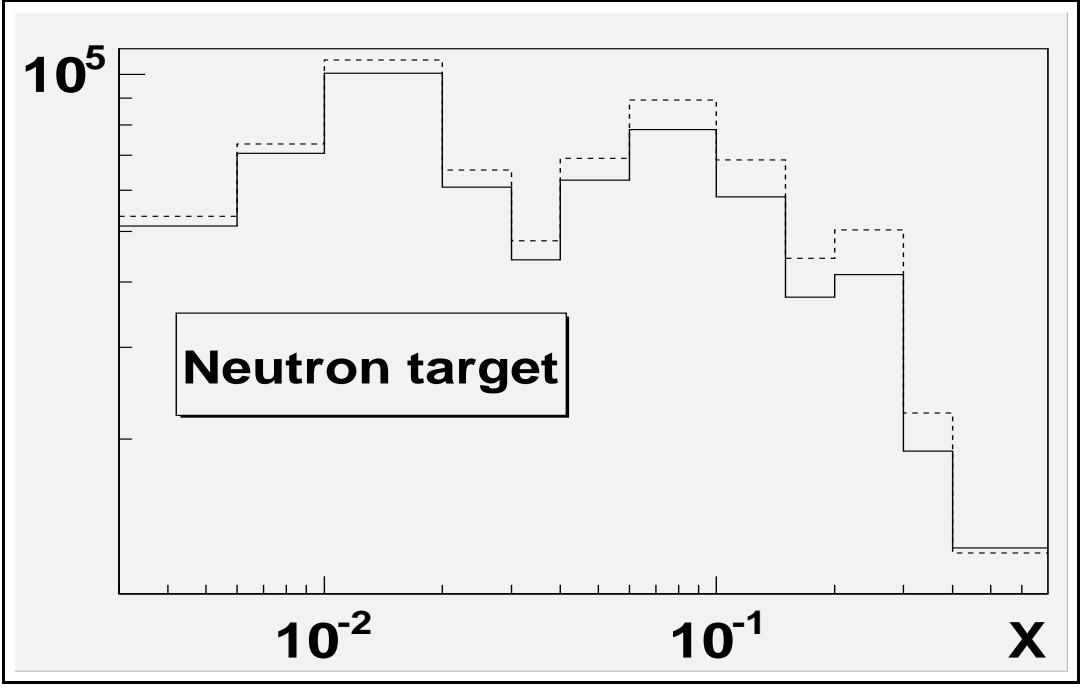

Figure 1: $N^{\pi^{+}}$and $N^{\pi^{-}}$obtained with PEPSI for neutron target. The dashed and solid lines correspond to $\pi^{+}$, and $\pi^{-}$productions, respectively.

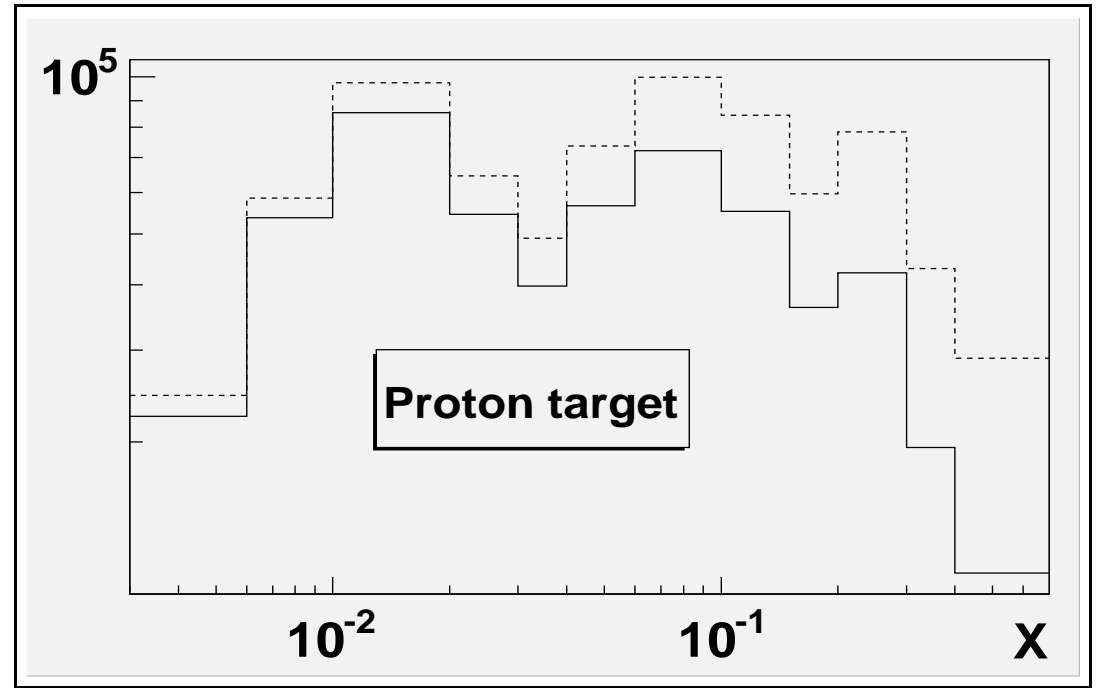

Figure 2: $N^{\pi^{+}}$and $N^{\pi^{-}}$obtained with PEPSI for proton target. The dashed and solid lines correspond to $\pi^{+}$, and $\pi^{-}$productions, respectively.

To be sure that concerning the strong asymmetry between $N_{p, d}^{\pi^{+}}$and $N_{p, d}^{\pi^{-}}$the PEPSI event generator we deal with strictly follows the real [14] physical picture, one can also compare what we have obtained with PEPSI Fig. 4 for the ratio of $N_{p, d}^{\pi^{+}}$and $N_{p, d}^{\pi^{-}}$with Fig. 10 in ref. [14].

Thus, the differences between the total counting rates $N^{\pi^{+}} \equiv N_{\uparrow \downarrow}^{\pi^{+}}+N_{\uparrow \uparrow}^{\pi^{+}}$and $N^{\pi^{-}} \equiv$ $N_{\uparrow \downarrow}^{\pi^{-}}+N_{\uparrow \uparrow}^{\pi^{-}}$are not small quantities ${ }^{15}$ for both proton and deutron targets, and, besides, increase

${ }^{15}$ Though in the vicinity $x_{B}=0$ the ratio $N^{\pi^{+}} / N^{\pi^{-}}$approaches unity (see Fig. 4), with the applied statistics $3 \cdot 10^{6}$ DIS events (absolutely real for HERMES and COMPASS - see below), the difference $N^{\pi^{+}}-N^{\pi^{-}}$entering the error significantly differs from zero even near the minimal value $x_{B}=0.003$ accessible to measurement. Indeed, in the first bin $0.003<x<0.006$ the PEPSI event generator gives for the proton target $N^{\pi^{+}} / N^{\pi^{-}}=$ 1.085 while $N^{\pi^{+}}-N^{\pi^{-}}=2985$, and for deutron target $N^{\pi^{+}} / N^{\pi^{-}}=1.053$ while $N^{\pi^{+}}-N^{\pi^{-}}=2020$. 


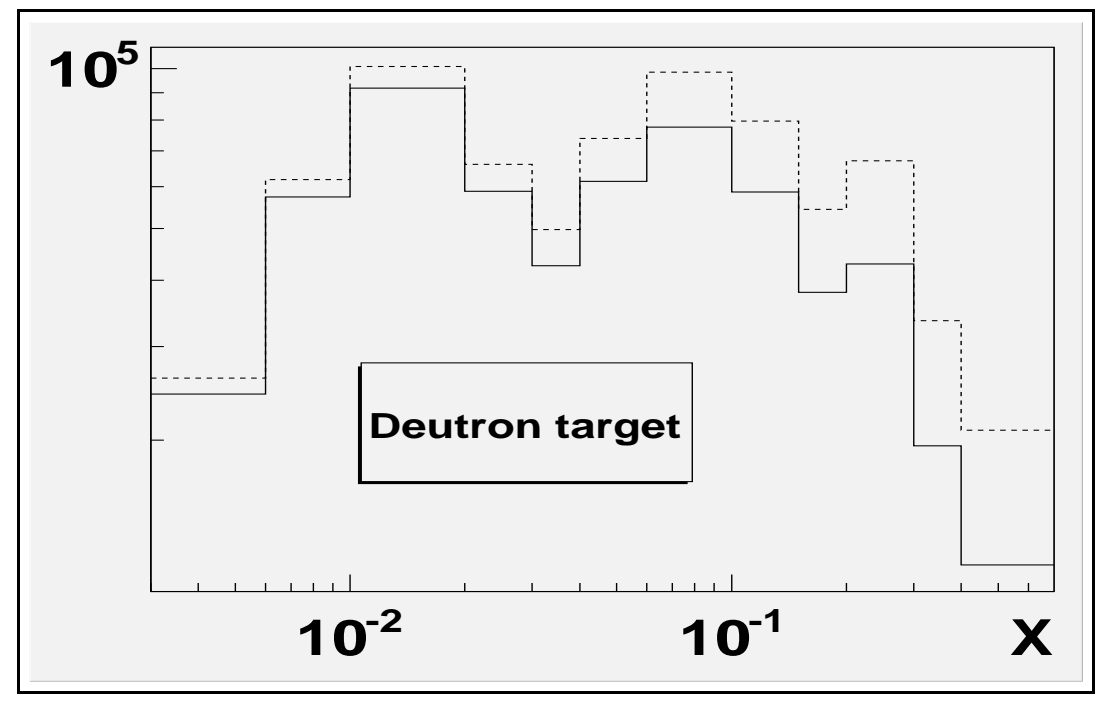

Figure 3: $N^{\pi^{+}}$and $N^{\pi^{-}}$obtained with PEPSI for deutron target. The dashed and solid lines correspond to $\pi^{+}$, and $\pi^{-}$productions, respectively.

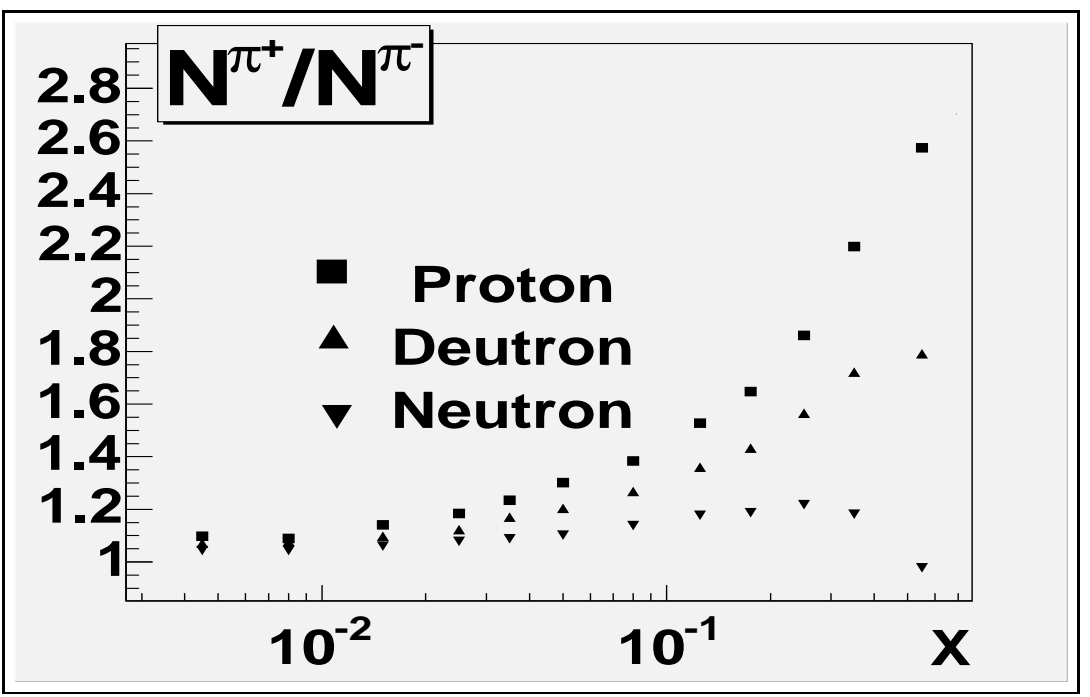

Figure 4: Ratios of $N^{\pi^{+}} \equiv N_{\uparrow \downarrow}^{\pi^{+}}+N_{\uparrow \uparrow}^{\pi^{+}}$and $N^{\pi^{-}} \equiv N_{\uparrow \downarrow}^{\pi^{-}}+N_{\uparrow \uparrow}^{\pi^{-}}$obtained with the polarized event generator PEPSI for the different targets. The picture is in good agreement with the respective EMC result - Fig. 10 in ref. [14.

with the statistics. As a result, the respective statistical errors turn out to be quite acceptable.

Let us illustrate this statement by a simple LO example. Using the GRSV2000LO(broken sea) [16] parametrization entering the PEPSI event generator as the input, we generate $3 \cdot 10^{6}$ DIS events with $E_{\mu}=160 \mathrm{GeV}^{2}$ (COMPASS kinematics). We then construct the "experimental" asymmetries together with their statistical errors using Eqs. (A.1) and (A.7) from the Appendix, respectively. These simulated asymmetries are compared with the theoretical ones given by Eqs. (3) - see Fig. 5. One can see that the errors on the simulated asymmetries are quite acceptable and that the simulated and theoretical asymmetries are in good agreement 


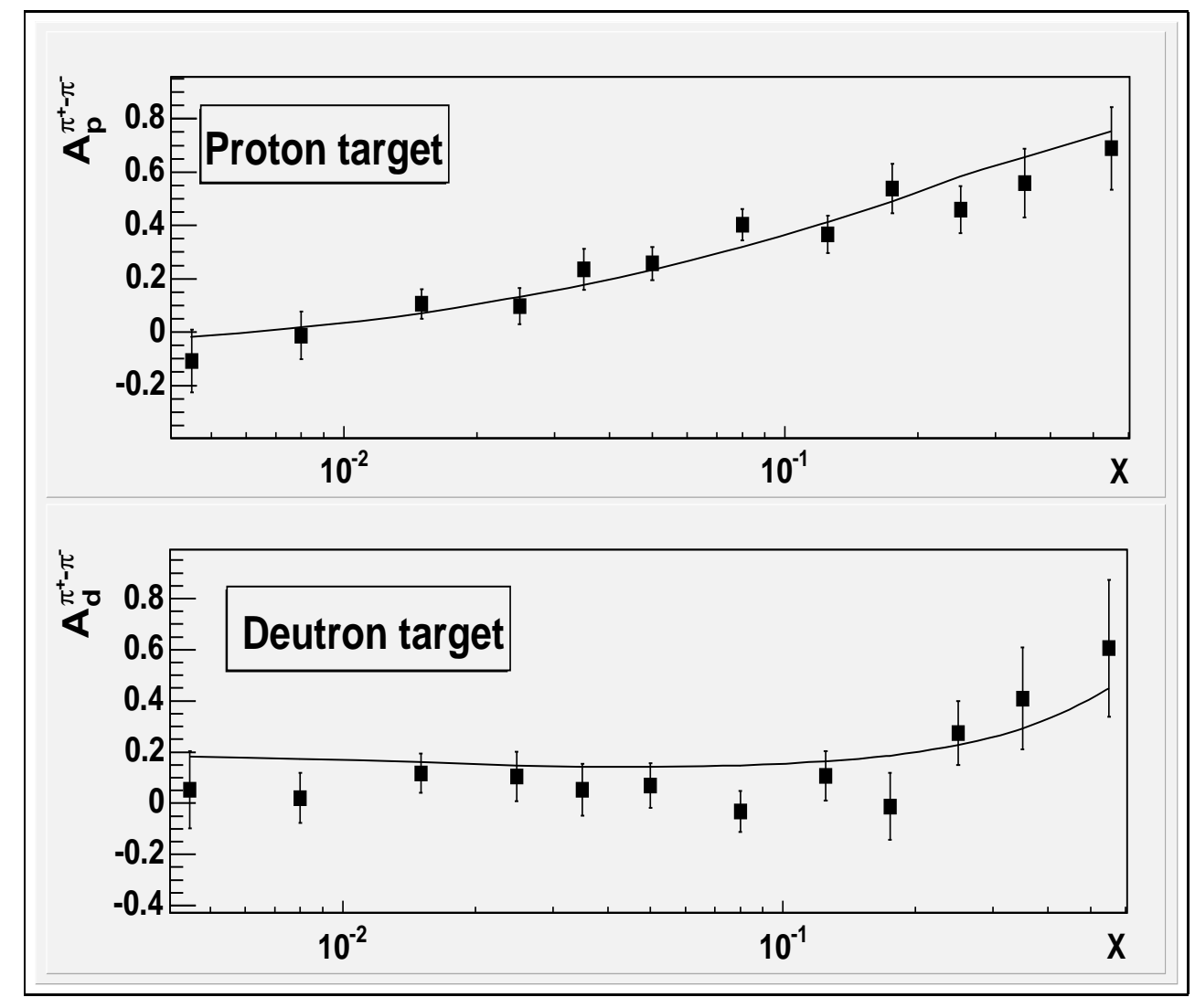

Figure 5: Simulated (squares) and theoretical pion difference asymmetries for proton and deutron targets. The solid lines correspond to the theoretical asymmetries obtained from Eq. (3) with GRSV2000LO(broken sea) parametrizations for the valence distributions.

within the errors. Furthermore, it is seen from Fig. 6 that the extracted valence ${ }^{16}$ distributions are also in good accordance with the respective input parametrizations.

\section{Testing of the NLO QCD extraction procedure}

\section{A. Broken sea scenario}

To perform the NLO QCD analysis, we first choose $^{17}$ the GRSV2000NLO(broken sea) [16] parametrization as an input. The conditions of simulations are presented in Table 1 and correspond to HERMES and ${ }^{18}$ SMC (COMPASS) kinematics. Let us stress that all the cuts in Table 1 are the standard cuts applied ${ }^{19}$ by SMC, HERMES and COMPASS. The statistics $3 \cdot 10^{6}$ in Table 1 is the total number of DIS events for both proton and deutron target and for both longitudinal polarizations. Since the statistical error on the pion difference asymmetry

\footnotetext{
${ }^{16}$ Namely the valence distributions are essential for what follows - see below.

${ }^{17}$ Note that at present the broken sea scenario is argued as the most probable one [16] (see also discussion on this subject at the beginning of this paper and Eq. (1)).

${ }^{18}$ Since the COMPASS muon beam energy $160 \mathrm{GeV}$ is almost the same as that of SMC, $190 \mathrm{GeV}$, the COMPASS low $x_{B}$ boundary achieved at the asymmetry measurements also should be about the same as the SMC 0.003 .

${ }^{19}$ For example, the important cut on invariant mass $W^{2}>10 \mathrm{GeV}^{2}$ is applied by these collaborations to exclude the events coming from the resonance region.
} 


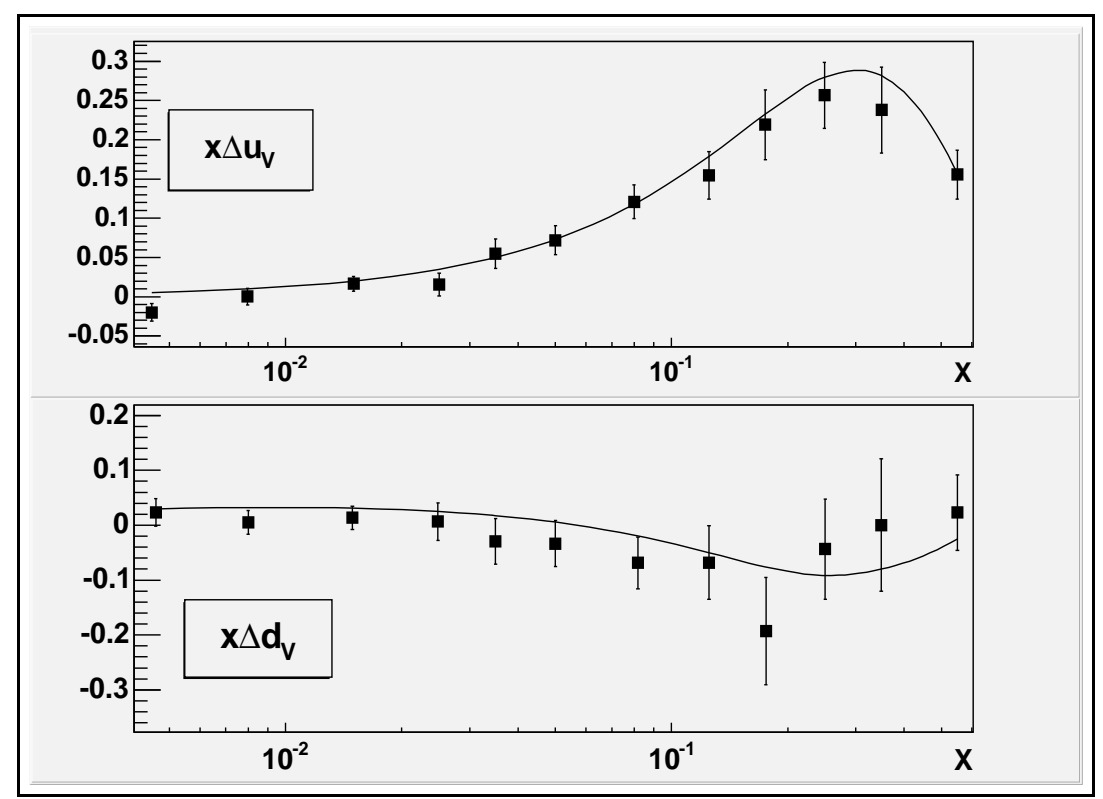

Figure 6: The reconstructed polarized valence distributions (squares). The solid lines correspond to the respective GRSV2000LO(broken sea) parametrizations.

depends on $N^{\pi^{+}}$and $N^{\pi^{-}}$, one need to know the respective semi-inclusive statistics - the total (for all available $x_{B}$ ) number of pions $N_{\text {tot }}^{\pi^{+}}$and $N_{\text {tot }}^{\pi^{-}}$corresponding to $1.5 \cdot 10^{6}$ DIS events for each target. With the all cuts indicated in Table 1, the PEPSI event generator gives

$\left.N_{\text {tot }}^{\pi^{+}}\right|_{\text {proton }}=551281,\left.N_{\text {tot }}^{\pi^{-}}\right|_{\text {proton }}=358654 ;\left.\quad N_{\text {tot }}^{\pi^{+}}\right|_{\text {deutron }}=526747,\left.N_{\text {tot }}^{\pi^{-}}\right|_{\text {deutron }}=383826$,

for $E_{l}=27 \mathrm{GeV}$, while

$\left.N_{\text {tot }}^{\pi^{+}}\right|_{\text {proton }}=582913,\left.N_{\text {tot }}^{\pi^{-}}\right|_{\text {proton }}=420709 ;\left.\quad N_{\text {tot }}^{\pi^{+}}\right|_{\text {deutron }}=559494,\left.N_{\text {tot }}^{\pi^{-}}\right|_{\text {deutron }}=447599$,

for $E_{l}=160 \mathrm{GeV}$. It is of importance that these numbers are absolutely realistic for HERMES (in 2000 HERMES already achieved for deutron target $N_{\text {tot }}^{\pi^{+}}=493492, N_{t o t}^{\pi^{-}}=402479$ - see Table 5.4 in ref. [17]) and even much less than expected by COMPASS (see COMPASS proposal [7, p. 90$)$.

Table 1: Simulation conditions. A and B correspond to HERMES and SMC (COMPASS) kinematics, respectively. Here $x_{B}$ and $x_{F}$ are the Bjorken and Feynman $x$ variables, respectively, $z_{h}=$ is the standard hadronic variable and $W$ is the invariant mass of the final hadronic state.

\begin{tabular}{|c|c|c|c|c|c|c|}
\hline Kinematics & $E_{\text {lepton }}$ & $x_{B}$ & $x_{F}$ & $z_{h}$ & $W^{2}$ & Events \\
\hline $\mathrm{A}$ & $27.5 \mathrm{GeV}$ & $0.023<x_{B}<0.6$ & $x_{F}>0.1$ & $z_{h}>Z=0.2$ & $W^{2}>10 \mathrm{GeV}^{2}$ & $3 \cdot 10^{6}$ \\
\hline $\mathrm{B}$ & $160 \mathrm{GeV}$ & $0.003<x_{B}<0.7$ & $x_{F}>0.1$ & $z_{h}>Z=0.2$ & $W^{2}>10 \mathrm{GeV}^{2}$ & $3 \cdot 10^{6}$ \\
\hline
\end{tabular}

To extract the quantities $\Delta_{1} u_{V}, \Delta_{1} d_{V}$ and, eventually, $\Delta_{1} \bar{u}-\Delta_{1} \bar{d}$ from the simulated asymmetries, one should first construct the difference asymmetries together with their statistical errors using Eqs. (A.1) and (A.7) from the Appendix, and then calculate the quantities $\mathcal{A}_{p(d)}^{\text {exp }}$ and $L_{1}-L_{2}$ entering Eqs. (20), (25). For $\mathcal{A}_{p}^{\text {exp }}$ one should use, instead of integral formula (21) 
the equation (and analogously for $\mathcal{A}_{d}^{\text {exp }}$ )

$$
\mathcal{A}_{p}^{e x p}=\left.\sum_{i=1}^{N_{\text {bins }}} \Delta x_{i} A_{p}^{\pi^{+}-\pi^{-}}\left(x_{i}\right)\right|_{Z}\left(4 u_{V}-d_{V}\right)\left(x_{i}\right) \int_{Z}^{1} d z_{h}\left[1+\otimes \frac{\alpha_{s}}{2 \pi} \tilde{C}_{q q} \otimes\right]\left(D_{1}-D_{2}\right),
$$

where $\Delta x_{i}$ is the $i-t h$ bin width. The parametrizations [18] for the fragmentation functions and [19] for unpolarized quark distributions are used. Note that here one should not use the usual "+"-prescription in the Wilson coefficients $C_{q q}$, but its generalization, the so-called "A"prescription [20. The calculation of $L_{1}, L_{2}$ is rather simple and can be done using any numerical method.

Let us introduce the additional notation $\Delta_{1}^{*} q_{V}=\int_{x_{\min }}^{x_{\max }} d x \Delta q_{V}$ and rewrite ${ }^{20} \mathrm{BSR}$ in the form (24) as

$$
\begin{array}{r}
\Delta_{1} \bar{u}-\Delta_{1} \bar{d}=\left[\Delta_{1}^{*} \bar{u}-\Delta_{1}^{*} \bar{d}\right]_{B S R}-\frac{1}{2} \int_{0}^{x_{\min }} d x\left(\Delta u_{V}-\Delta d_{V}\right), \\
{\left[\Delta_{1}^{*} \bar{u}-\Delta_{1}^{*} \bar{d}\right]_{B S R}=\frac{1}{2}\left|\frac{g_{A}}{g_{V}}\right|-\frac{1}{2}\left(\Delta_{1}^{*} u_{V}-\Delta_{1}^{*} d_{V}\right),}
\end{array}
$$

where $x_{\min }$ and $x_{\max }$ are the boundary points of the available Bjorken $x$ region. It is obvious that dealing with the restricted available Bjorken $x$ regions, one can directly extract from the measured difference asymmetries namely the quantities $\Delta_{1}^{*} u_{V}, \Delta_{1}^{*} d_{V}$ and $\left[\Delta_{1}^{*} \bar{u}-\Delta_{1}^{*} \bar{d}\right]_{B S R}$, while the "tail" contributions $\int_{0}^{x_{\min }} d x \Delta u_{V}, \int_{0}^{x_{\min }} d x \Delta d_{V}$ and $\frac{1}{2} \int_{0}^{x_{\min }} d x\left(\Delta u_{V}-\Delta d_{V}\right)$ should be studied separately, applying the proper extrapolation procedure (see below).

The results on $\Delta_{1}^{*} u_{V}, \Delta_{1}^{*} d_{V}$ and $\left[\Delta_{1}^{*} \bar{u}-\Delta_{1}^{*} \bar{d}\right]_{B S R}$ extracted from the simulated difference asymmetries using the presented NLO procedure, are given in Table 2.

Table 2: GRSV2000NLO(broken sea) parametrization. Results on $\Delta_{1}^{*} u_{V}, \Delta_{1}^{*} d_{V}$ and $\left[\Delta_{1}^{*} \bar{u}-\Delta_{1}^{*} \bar{d}\right]_{B S R}$ extracted from the simulated difference asymmetries applying the proposed NLO procedure.

\begin{tabular}{|c|c|c|c|c|}
\hline Kinematics & $Q_{\text {mean }}^{2}$ & $\Delta_{1}^{*} u_{V}$ & $\Delta_{1}^{*} d_{V}$ & {$\left[\Delta_{1}^{*} \bar{u}-\Delta_{1}^{*} d\right]_{B S R}$} \\
\hline $\mathrm{A}$ & $2.4 \mathrm{GeV}^{2}$ & $0.585 \pm 0.017$ & $-0.147 \pm 0.037$ & $0.268 \pm 0.020$ \\
\hline $\mathrm{B}$ & $7.0 \mathrm{GeV}^{2}$ & $0.602 \pm 0.032$ & $-0.110 \pm 0.080$ & $0.278 \pm 0.040$ \\
\hline
\end{tabular}

It is obvious that to be valid, the extraction procedure, being applied to the simulated asymmetries should yield results maximally close to the ones obtained directly from the parametrization entering the generator as an input. The results for the respective parametrization functions integrated over the total $0<x<1$ region in Bjorken $x$ and over the regions $0.023<x<0.6$ (HERMES [3] kinematics) and $0.003<x<0.7$ (COMPASS kinematics) are presented by the Table 3.

Let us now compare the results from Tables 2 and 3.

First of all notice that contrary to the actual experiment conditions, the simulations give the possibility to check the validity of the extraction method comparing the results of the extraction from the simulated asymmetries with an exact answer. Namely, in our case this is the integral over the total region of the difference of the parametrizations for $\Delta \bar{u}$ and $\Delta \bar{d}$ entering the generator as an input:

$$
\left[\Delta_{1} \bar{u}-\Delta_{1} \bar{d}\right]_{e x a c t} \simeq\left[\Delta_{1}^{*} \bar{u}-\Delta_{1}^{*} \bar{d}\right]_{25}=\int_{0.0001}^{0.99} d x[\Delta \bar{u}-\Delta \bar{d}]_{\text {parametrization }}=0.310
$$

\footnotetext{
${ }^{20}$ As usual, we neglect contributions to $\Delta_{1} q$ from the unmeasured large $x_{B}$ region $0.6(0.7)<x_{B}<1$ because their upper limits given by the unpolarized distributions are very small there - see $[3][6]$.
} 
Table 3: Results on $\Delta_{1}^{*} u_{V}, \Delta_{1}^{*} d_{V}, \Delta_{1}^{*} \bar{u}-\Delta_{1}^{*} \bar{d}$ and $\left[\Delta_{1}^{*} \bar{u}-\Delta_{1}^{*} \bar{d}\right]_{B S R}$ obtained from integration of the GRSV2000NLO (broken sea) parametrization of the quark distributions over the total and experimentally available Bjorken $x$ regions. The fifth column is obtained by direct integration of the respective parametrizations. The sixth column is obtained using BSR and the parametrizations for the valence distributions.

\begin{tabular}{|c|c|c|c|c|c|}
\hline$x_{B}$ & $Q^{2}$ & $\Delta_{1}^{*} u_{V}$ & $\Delta_{1}^{*} d_{V}$ & $\Delta_{1}^{*} \bar{u}-\Delta_{1}^{*} d$ & {$\left[\Delta_{1}^{*} \bar{u}-\Delta_{1}^{*} d\right]_{B S R}$} \\
\hline $0.0001<x_{B j}<0.99$ & $2.4 \mathrm{GeV}^{2}$ & 0.605 & -0.031 & 0.310 & 0.315 \\
\hline $0.023<x_{B j}<0.6$ & $2.4 \mathrm{GeV}^{2}$ & 0.569 & -0.114 & 0.170 & 0.292 \\
\hline $0.0001<x_{B j}<0.99$ & $7.0 \mathrm{GeV}^{2}$ & 0.604 & -0.032 & 0.309 & 0.315 \\
\hline $0.003<x_{B j}<0.7$ & $7.0 \mathrm{GeV}^{2}$ & 0.598 & -0.065 & 0.262 & 0.302 \\
\hline
\end{tabular}

where symbol $[\ldots]_{n m}$ denotes the $n$-th line and $m$-th column of Table 3.

The second point is that the integral taken directly (without using BSR) from the $\Delta \bar{u}$ and $\Delta \bar{d}$ parametrization difference over the available to HERMES region is almost two times less than the exact answer (30):

$$
\left[\Delta_{1}^{*} \bar{u}-\Delta_{1}^{*} \bar{d}\right]_{35}=\int_{0.023}^{0.6} d x[\Delta \bar{u}-\Delta \bar{d}]_{\text {parametrization }}=0.170 .
$$

This is a direct indication that the HERMES interval in Bjorken $x$ is too narrow ${ }^{21}$ to extract the quantity $\Delta_{1} \bar{u}-\Delta_{1} \bar{d}$ we are interested in directly.

However, there is a possibility to avoid this trouble and essentially improve the analysis on $\Delta_{1} \bar{u}-\Delta_{1} \bar{d}$ even with the narrow HERMES $x_{B}$ region, applying BSR for $\Delta_{1} \bar{u}-\Delta_{1} \bar{d}$ extraction. Indeed, applying Eq. (29) to the HERMES $x_{B}$ region, considering that $\left|g_{A} / g_{V}\right|=1.2670 \pm$ 0.0035 and calculating the integrals of the valence quark parametrizations over the region $0.023<x<0.6$, one gets

$$
\left[\Delta_{1}^{*} \bar{u}-\Delta_{1}^{*} \bar{d}\right]_{36}^{B S R}=0.292,
$$

and this result (contrary to Eq. (31)) is in good agreement with the exact one, Eq. (301).

The reason of this good agreement of Eq. (32) with the exact answer Eq. (30) is that, contrary to the sea distributions, the valence distributions gather far from the low boundary $x_{B}=0$ (see, for example [14] and references therein).

Thus, this exercise with the integrals of the parametrization functions shows that, at least within the broken sea scenario, the application of Eq. (24) for $\Delta_{1} \bar{u}-\Delta_{1} \bar{d}$ extraction could give a reliable result on this quantity even with the narrow HERMES $x_{B}$ region. Namely, one should first extract in the accessible $x_{B}$ region the truncated moments of the valence distributions, and only then get the quantity $\left[\Delta_{1}^{*} \bar{u}-\Delta_{1}^{*} \bar{d}\right]_{B S R}$ applying Eq. (29).

One can also compare elements 55 and 56 from Table 3 corresponding to the SMC (COMPASS) Bjorken $\mathrm{x}$ region with the exact answer, element 45 from the Table 3. It is seen that even though the integral over the experimentally available region taken directly of the sea parametrization difference is now much closer to the exact answer, the application of BSR instead of direct extraction significantly improves the situation even for this much wider $x_{B}$ region.

Returning now to the proposed NLO extraction procedure, let us recall that the application of BSR in the form (24) (see derivation of Eq. (25)) is one of the essential elements of the

\footnotetext{
${ }^{21}$ Note that the proposed NLO extraction procedure has nothing to do with that problem - we just compare the integrals of the parametrization over the different Bjorken $x$ regions.
} 
procedure. Comparing the result of Table 2 on $\left[\Delta_{1}^{*} \bar{u}-\Delta_{1}^{*} \bar{d}\right]_{B S R}$ obtained from the simulated asymmetries with the HERMES kinematics (element 25 from the Table 2) with both Eqs. (30) and (32), one can see that they are in good agreement with each other. Besides, comparing the results of Tables 2 and 3 for the COMPASS kinematics, one can see that the results on reconstructed $\Delta_{1}^{*} u_{V}, \Delta_{1}^{*} d_{V}$ and $\Delta_{1}^{*} \bar{u}-\Delta_{1}^{*} \bar{d}$ are in still good agreement with the respective quantities obtained by direct integration of the input parametrization over both the total $0<$ $x_{B}<1$ and experimentally available $0.003<x_{B}<0.7$ regions.

It is also of importance that even without BSR application, the moments of the valence distributions (interesting in themselves) extracted in NLO in the accessible $x_{B}$ regions, are in a good agreement with the input parametrization for both HERMES and COMPASS kinematics.

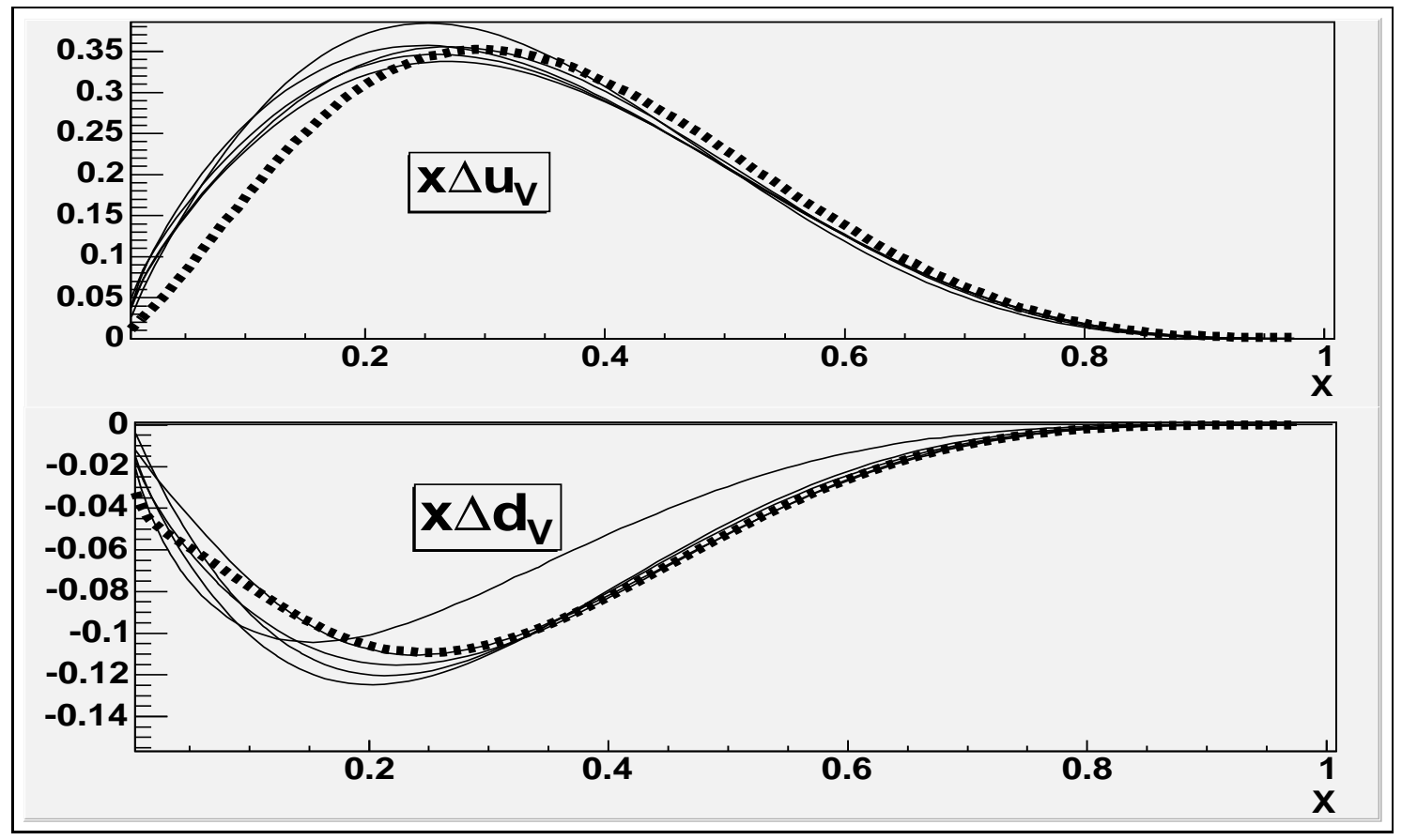

Figure 7: Polarized valence distributions given by the different parametrizations with the symmetric and weakly broken sea. Solid lines corresponds to the purely symmetric parametrizations from refs. [16] and 22, while the dashed line corresponds to parametrization FS2000 (set iin ref. [10]) with weakly broken sea. All of these parametrizations demonstrate quite similar behavior.

\section{B. Symmetric sea scenario}

Until now we dealt with the broken sea scenario (which seems to be the most probable one see footnote 17). However, one, certainly, should also investigate an alternative opportunity the symmetric sea scenario. Notice that all the known parametrizations with the symmetric or weakly broken ${ }^{22}$ polarized sea essentially differ from the only presently known parametrization

\footnotetext{
${ }^{22}$ The only such parametrization which we know is FS2000 parametrization [10]. $\Delta_{1} \bar{u}-\Delta_{1} \bar{d}<0.1$ within this parametrization, and with respect to the valence distribution it behaves quite analogously to the purely symmetric parametrizations - See Fig. 7. At the same time the behavior of the parametrization with the strongly broken sea, GRSV2000 (broken sea), is absolutely different - contrary to the parametrizations with the symmetric or weakly broken sea, $\Delta d_{V}$ changes the sign at small $x_{B}$ where the contribution of the sea quarks become dominant.
} 
with the strongly broken sea, GRSV2000 (broken sea), we dealt with previously. However, they rather little differ from each other - see Fig. 7. So, for self-consistence, we again choose GRSV2000NLO (but with the symmetric sea) parametrization as an alternative input. The respective analysis is presented in Table 4.

Table 4: The upper part presents the results on $\Delta_{1}^{*} u_{V}, \Delta_{1}^{*} d_{V}$ and $\left[\Delta_{1}^{*} \bar{u}-\Delta_{1}^{*} \bar{d}\right]_{B S R}$ obtained from integration of the GRSV2000NLO(symmetric sea) [16] parametrization. The lower part presents the results on $\Delta_{1}^{*} u_{V}$, $\Delta_{1}^{*} d_{V}$ and $\left[\Delta_{1}^{*} \bar{u}-\Delta_{1}^{*} \bar{d}\right]_{B S R}$ extracted from the simulated difference asymmetries applying the proposed NLO procedure with parametrization GRSV2000NLO(symmetric sea) entering the generator as the input.

\begin{tabular}{|c|c|c|c|c|}
\hline$x_{B}$ & $Q^{2}$ & $\Delta_{1}^{*} u_{V}$ & $\Delta_{1}^{*} d_{V}$ & {$\left[\Delta_{1}^{*} \bar{u}-\Delta_{1}^{*} d\right]_{B S R}$} \\
\hline $0.023<x_{B j}<0.6$ & $2.4 \mathrm{GeV}^{2}$ & 0.749 & -0.276 & 0.121 \\
\hline $0.003<x_{B j}<0.7$ & $7.0 \mathrm{GeV}^{2}$ & 0.866 & -0.320 & 0.041 \\
\hline $0.0001<x_{B j}<0.99$ & $2.4 \mathrm{GeV}^{2}$ & 0.916 & -0.339 & 0.006 \\
\hline $0.0001<x_{B j}<0.99$ & $7.0 \mathrm{GeV}^{2}$ & 0.914 & -0.339 & 0.007 \\
\hline \hline Kinematics & $Q_{\text {mean }}^{2}$ & $\Delta_{1}^{*} u_{V}$ & $\Delta_{1}^{*} d_{V}$ & $\Delta_{1}^{*} \bar{u}-\Delta_{1}^{*} d$ \\
\hline $\mathrm{A}$ & 2.4 & $0.736 \pm 0.017$ & $-0.310 \pm 0.037$ & $0.111 \pm 0.020$ \\
\hline $\mathrm{B}$ & $7.0 \mathrm{GeV}^{2}$ & $0.842 \pm 0.032$ & $-0.300 \pm 0.069$ & $0.063 \pm 0.038$ \\
\hline
\end{tabular}

Let us analyze the results from Table 4. First, one can see that for both A and B kinematics, the results of reconstruction in the accessible $x_{B}$ region of the all presented in this table quantities are again in good agreement with the input parametrization. Thus, the analysis performed within the symmetric sea scenario again confirms that the proposed NLO extraction procedure satisfies the main criterion of validity - to reconstruct the quark moments in the experimentally available $x_{B}$ region.

On the other hand, performing the reconstruction of the entire quantity $\Delta_{1} \bar{u}-\Delta_{1} \bar{d}$, one, certainly, should not roughly put it to $\Delta_{1}^{*} \bar{u}-\Delta_{1}^{*} \bar{d}$. It is necessary to carefully estimate the unmeasured "tail" $\frac{1}{2} \int_{0}^{x_{\min }} d x\left(\Delta u_{V}-\Delta d_{V}\right)$ entering Eq. (28), especially dealing with so narrow Bjorken $x$ region as the HERMES one. It is clearly seen from the Table 4 where the result on $\Delta_{1}^{*} \bar{u}-\Delta_{1}^{*} \bar{d}$ is quite close to the exact answer, zero, for the COMPASS $x_{B}$ region, but in the case of HERMES kinematics it indicates rather essential deviation from the zero value.

\section{Low $x_{B}$ uncertainties}

Let us stress that the problem of the unmeasured "tail" estimation is the common and long staying problem which, however, in any case should be somehow solved if we wish definitely answer the question is the sea symmetric or not. Nowadays the state of art is such that the polarized SIDIS experiments use the only method of the low $x_{B}$ contribution estimations (see, for example, [3], [7]): the proper fit to the obtained data on $\Delta q$ is performed with the subsequent extrapolation of the fitting function to unavailable low $x_{B}$. On the other hand, the low $x_{B}$ "tails" of all the existing parametrizations on $\Delta q$ are obtained using a quite analogous procedure. Namely, the parametrization on $\Delta q$ is extracted in the accessible $x_{B}$ region from the fit to the measured inclusive asymmetries and/or structure functions and then is extrapolated to low $x_{B}$. It is also of importance that the degree of the reliability of the low $x_{B}$ estimations applied in the existing parametrizations increases due to that all the parametrizations are constructed in the strict accordance with the sum rules on $a_{3}$ and $a_{8}$ nonsinglet combinations. Besides, the constructed parametrizations meet the requirement of agreement with the existing DIS data on $\Gamma_{1}^{p}$ and $\Gamma_{1}^{n}[21]$. 
Table 5: Low- $x$ contributions to $\frac{1}{2}\left(\Delta_{1} u_{V}-\Delta_{1} d_{V}\right)$ for the different NLO parametrizations.

\begin{tabular}{|c|c|c|}
\hline \multirow{2}{*}{ NLO parametrization } & \multicolumn{2}{|c|}{$\int_{0}^{x_{\min }} d x\left(\Delta u_{V}-\Delta d_{V}\right) / 2$} \\
\cline { 2 - 3 } & $x_{\min }=0.023, Q^{2}=2.4 \mathrm{GeV}^{2}$ & $x_{\min }=0.003, Q^{2}=7.0 \mathrm{GeV}^{2}$ \\
\hline GRSV2000 (broken sea) & -0.035 & -0.016 \\
\hline \hline GRSV2000 (symmetric sea) & 0.110 & 0.033 \\
\hline FS2000 (i+) & 0.104 & 0.036 \\
\hline FS2000 (i-) & 0.080 & 0.031 \\
\hline LSS2001 & 0.098 & 0.032 \\
\hline AAC2000 & 0.116 & 0.046 \\
\hline AAC2003 & 0.127 & 0.055 \\
\hline
\end{tabular}

So, we propose to perform the respective estimation of the quantity $\int_{0}^{x_{\min }} d x\left(\Delta u_{V}-\Delta d_{V}\right)$ using the maximal number of the latest available NLO parametrizations. The results are presented in the Table 5, where the parametrizations from the refs. [10], [16] and 22] are used.

Looking at the Table 5 one can conclude that for the HERMES $x_{B}$ region

$$
\frac{1}{2} \int_{0}^{0.023} d x\left|\left(\Delta u_{V}-\Delta d_{V}\right)\right| \lesssim 0.13
$$

while for the COMPASS $x_{B}$ region the upper boundary is approximately twice as less:

$$
\frac{1}{2} \int_{0}^{0.003} d x\left|\left(\Delta u_{V}-\Delta d_{V}\right)\right| \lesssim 0.06 \text {. }
$$

Notice that one can estimate only absolute value of $\int_{0}^{x_{\min }} d x\left(\Delta u_{V}-\Delta d_{V}\right)$, because we do not know which scenario (symmetric or not) is realized in nature. For example, the well known broken sea parametrization GRSV2000 gives the negative sign for this quantity while all the symmetric sea parametrizations give the positive sign. It is also seen that the restrictions (33), (34) based on the results of Table 5 are rather strong. For safety, we deliberately overestimate the upper boundaries choosing the largest numbers from the Table 5 instead of performing the averaging procedure over all used parametrizations (just as it was done by SMC [6]). Notice also that the restriction (34) is consistent with the respective estimation made by SMC (see Table 5 in ref. [6]), whereas the upper boundary given by Eq. (33) is in four times larger than the HERMES estimation ${ }^{23}$.

Thus, to extract the entire quantity $\Delta_{1} \bar{u}-\Delta_{1} \bar{d}$ we propose to include the upper boundaries on $\frac{1}{2} \int_{0}^{x_{\min }} d x\left(\Delta u_{V}-\Delta d_{V}\right)$ given by the inequalities (33) and (34) into the respective systematical errors, so that the additional low $x_{B}$ contributions into the systematical errors of HERMES and COMPASS look as

$$
\begin{aligned}
\left.\delta_{\text {low } x}\right|_{\text {HERMES }} & = \pm 0.13, \\
\left.\delta_{\text {low } x}\right|_{\text {COMPASS }} & = \pm 0.06 .
\end{aligned}
$$

Certainly, Eqs. (35) (36) should not be considered as some strict estimations. This is just an attempt roughly but with all possible precautions to estimate could HERMES and (or) COMPASS under their real conditions answer the question is the sea broken or not.

\footnotetext{
${ }^{23}$ Notice that it is dangerous to use the simple Regge parametrization for extrapolation in the all rather large $x_{B}$ region unavailable to HERMES. The obtained in this way estimations [3] $\int_{0}^{0.023} d x \Delta u_{V} \simeq 0.03$, $\int_{0}^{0.023} d x \Delta d_{V} \simeq-0.03$ seem to be rather underestimated (see discussion on this subject in ref. [2]).
} 


\section{Discussion and conclusion}

With the (rather overestimated) uncertainties given by Eqs. (35), (36) it is quite possible that HERMES would not see within the total error that $\Delta_{1} \bar{u}-\Delta_{1} \bar{d} \neq 0$, if this quantity happens too small in reality (for example, about 0.2). However, if this quantity will be about 0.3 (as it assumed by GRSV2000 broken sea parametrization) and higher, it could be still possible to see this quantity even with the HERMES $x_{B}$ region. On the other hand, it is seen from the Tables 2, 3, 4 and Eq. (36) that the COMPASS $x_{B}$ region could allow to catch even small difference (if any) between $\Delta_{1} \bar{u}$ and $\Delta_{1} \bar{d}$.

In any case, analyzing such a tiny quantity as $\Delta_{1} \bar{u}-\Delta_{1} \bar{d}$ it is very desirable to perform a combined analysis with both HERMES and COMPASS data. For example, having at one's disposal data on the difference asymmetries in the accessible to HERMES $x_{B}$ region, one could involve in the analysis on $\Delta_{1} \bar{u}-\Delta_{1} \bar{d}$ the respective COMPASS data from the region $0.003<x_{B}<0.023$. The point is that a high statistics, especially at low $x_{B}$ is claimed as one of the COMPASS advantages [7] as compared with SMC and HERMES experiments.

Thus, we have tested the proposed NLO QCD extraction procedure performing the simulations corresponding to both the broken and symmetric sea scenarios. This analysis confirms that the procedure meets the main requirement: to reconstruct the quark moments in the accessible to measurement $x_{B}$ region. On the other hand, even with the overestimated low $x_{B}$ uncertainty (35), one can conclude that the question is $\Delta_{1} \bar{u}-\Delta_{1} \bar{d}$ equal to zero or not could be answered even with the HERMES kinematics in the case of strongly asymmetric polarized sea. In any case, the situation is much better with the available to COMPASS $x_{B}$ region.

\section{Acknowledgments}

The authors are grateful to R. Bertini, M. P. Bussa, O. Denisov, O. Gorchakov, A. Efremov, N. Kochelev, A. Korzenev, A. Kotzinian, V. Krivokhizhin, E. Kuraev, A. Maggiora, A. Nagaytsev, A. Olshevsky, G. Piragino, G. Pontecorvo, J. Pretz, I. Savin and O. Teryaev, for fruitful discussions.

\section{Appendix}

Calculating the asymmetries given by Eq. (12) together with their statistical errors, one should have in mind that, contrary to SMC and COMPASS experiments, in the HERMES conditions the quantities $N_{\uparrow \uparrow(\uparrow \downarrow)}^{\pi^{+}\left(\pi^{-}\right)}$entering Eq. (12) are not the pure counting rates, but the counting rates multiplied $^{24}$ by the respective luminosities [3]. Thus, in general case, one should use instead of Eq. (12) the equation ${ }^{25}$ :

$$
A_{p(n, d)}^{\pi^{+}-\pi^{-}}=\frac{1}{D}\left[\frac{\left(N_{\uparrow \downarrow}^{\pi^{+}}-N_{\uparrow \downarrow}^{\pi-}\right) L_{\uparrow \uparrow}-\left(N_{\uparrow \uparrow}^{\pi^{+}}-N_{\uparrow \uparrow}^{\pi^{-}}\right) L_{\uparrow \downarrow}}{\left(N_{\uparrow \downarrow}^{\pi^{+}}-N_{\uparrow \downarrow}^{\pi-}\right) L_{\uparrow \uparrow}+\left(N_{\uparrow \uparrow}^{\pi^{+}}-N_{\uparrow \uparrow}^{\pi^{-}}\right) L_{\uparrow \downarrow}}\right],
$$

where luminosities $L_{\uparrow \uparrow(\uparrow \downarrow)}$ are defined as

$$
L_{\uparrow \uparrow(\uparrow \downarrow)}=(n \Phi)_{\uparrow \uparrow(\uparrow \downarrow)},
$$

\footnotetext{
${ }^{24}$ The cancellation of the luminosities is possible only with the special target setup, like the SMC and COMPASS ones [6, 7].

${ }^{25}$ Here the beam and target are assumed to be ideal which means that $P_{B}=P_{T}=f=1$. Namely this assumption is adopted in the PEPSI event generator [13] we use for simulations.
} 
where $n$ is th area density of the nucleons in the target and $\Phi$ is the beam flux. Within the paper we do not study the specific peculiarities of the different experimental setups and deal only with the event generator where the acceptance $a$ is equal to unity, so that

$$
N_{\uparrow \downarrow(\uparrow \uparrow)}=(n \Phi)_{\uparrow \downarrow(\uparrow \uparrow)} a \sigma_{\uparrow \downarrow(\uparrow \uparrow)} \rightarrow(n \Phi)_{\uparrow \downarrow(\uparrow \uparrow)} \sigma_{\uparrow \downarrow(\uparrow \uparrow)}
$$

Thus, Eq. A.2 for luminosities is rewritten in the following, suitable for simulations, form

$$
L_{\uparrow \uparrow(\uparrow \downarrow)}=N_{\uparrow \uparrow(\uparrow \downarrow)} /\left(\sigma_{\uparrow \uparrow(\uparrow \downarrow)}\right),
$$

where $N_{\uparrow \uparrow(\uparrow \downarrow)}$ are the numbers of inclusive events and $\sigma_{\uparrow \uparrow(\uparrow \downarrow)}$ are the inclusive cross-sections automatically calculated by PEPSI (see ref. [13]) for given sets of the kinematic conditions.

Choosing as the variables in Eq. A.1 the set $N_{\uparrow \downarrow}^{\pi^{+}}, N_{\uparrow \downarrow}^{\pi^{-}}, N_{\uparrow \uparrow}^{\pi^{+}}, N_{\uparrow \uparrow}^{\pi^{-}}$and using the general formula (see, for example, [23]) for the statistical error on the function $F$ of variables $x_{1}, x_{2}, \ldots$

$$
\delta^{2}\left(F\left(x_{1}, x_{2}, \ldots\right)\right)=\left(\frac{\partial F}{x_{1}}\right)^{2} \delta^{2}\left(x_{1}\right)+\left(\frac{\partial F}{x_{2}}\right)^{2} \delta^{2}\left(x_{2}\right)+2\left(\frac{\partial F}{x_{1}}\right)\left(\frac{\partial F}{x_{2}}\right) \operatorname{cov}\left(x_{1}, x_{2}\right)+\ldots
$$

one gets

$$
\begin{array}{r}
\delta^{2}\left(A_{p(n, d)}^{\pi^{+}-\pi^{-}}\right)=\frac{1}{D^{2} Y^{4}}\left\{(Y-X)^{2} L_{\uparrow \uparrow}^{2}\left[\delta^{2}\left(N_{\uparrow \downarrow}^{\pi^{+}}\right)+\delta^{2}\left(N_{\uparrow \downarrow}^{\pi^{-}}\right)-2 \operatorname{cov}\left(N_{\uparrow \downarrow}^{\pi^{+}}, N_{\uparrow \downarrow}^{\pi^{-}}\right)\right]\right. \\
\left.+(Y+X)^{2} L_{\uparrow \downarrow}^{2}\left[\left(\delta^{2} N_{\uparrow \uparrow}^{\pi^{+}}\right)+\delta^{2}\left(N_{\uparrow \uparrow}^{\pi^{-}}\right)\right]-2 \operatorname{cov}\left(N_{\uparrow \uparrow}^{\pi^{+}}, N_{\uparrow \uparrow}^{\pi^{-}}\right)\right\},
\end{array}
$$

where $X$ and $Y$ are the numerator and denominator in the square brackets in Eq. (A.1) . If the distributions of hadrons $N_{\uparrow \downarrow}^{\pi^{+}}, N_{\uparrow \downarrow}^{\pi^{-}}, N_{\uparrow \uparrow}^{\pi^{+}}, N_{\uparrow \uparrow}^{\pi^{-}}$are Poissonian (low multiplicities $n^{+}$, $n^{-}$- see [23] $): \delta\left(N_{\uparrow \uparrow(\uparrow \downarrow)}^{\pi^{+}\left(\pi^{-}\right)}\right)=\sqrt{N_{\uparrow \uparrow(\uparrow \downarrow)}^{\pi^{+}\left(\pi^{-}\right)}}$, then one can neglect in Eq. (A.6) the covariations $\operatorname{cov}\left(N_{\uparrow \uparrow}^{\pi^{+}}, N_{\uparrow \uparrow}^{\pi^{-}}\right)$and $\operatorname{cov}\left(N_{\uparrow \downarrow}^{\pi^{+}}, N_{\uparrow \downarrow}^{\pi^{-}}\right)$with a result:

$$
\delta^{2}\left(A_{p(n, d)}^{\pi^{+}-\pi^{-}}\right)=\frac{4 L_{\uparrow \uparrow}^{2} L_{\uparrow \downarrow}^{2}}{D^{2}} \frac{\left(N_{\uparrow \uparrow}^{\pi^{+}}-N_{\uparrow \uparrow}^{\pi^{-}}\right)^{2}\left[N_{\uparrow \downarrow}^{\pi^{+}}+N_{\uparrow \downarrow}^{\pi^{-}}\right]+\left(N_{\uparrow \downarrow}^{\pi^{+}}-N_{\uparrow \downarrow}^{\pi^{-}}\right)^{2}\left[N_{\uparrow \uparrow}^{\pi^{+}}+N_{\uparrow \uparrow}^{\pi^{-}}\right]}{\left[\left(N_{\uparrow \downarrow}^{\pi^{+}} L_{\uparrow \uparrow}+N_{\uparrow \uparrow}^{\pi^{+}} L_{\uparrow \downarrow}\right)-\left(N_{\uparrow \downarrow}^{\pi^{-}} L_{\uparrow \uparrow}+N_{\uparrow \uparrow}^{\pi^{-}} L_{\uparrow \downarrow}\right)\right]^{4}} .
$$

Operating absolutely analogously, one gets for the error on the usual spin asymmetry the equation

$$
\delta^{2}\left(A_{p(n, d)}^{\pi^{+}}\right)=\frac{1}{D^{2}} \frac{4 L_{\uparrow \downarrow}^{2} L_{\uparrow \uparrow}^{2}\left(N_{\uparrow \downarrow}^{\pi^{+}}+N_{\uparrow \uparrow}^{\pi^{+}}\right) N_{\uparrow \downarrow}^{\pi^{+}} N_{\uparrow \uparrow}^{\pi^{+}}}{\left(N_{\uparrow \downarrow}^{\pi^{+}} L_{\uparrow \uparrow}+N_{\uparrow \uparrow}^{\pi^{+}} L_{\uparrow \downarrow}\right)^{4}} .
$$

Notice that namely the equation (A.8) for the statistical error on the usual semi-inclusive asymmetry was used by HERMES [17.

For the COMPASS experiment $L_{\uparrow \uparrow}=L_{\uparrow \downarrow}$ and Eq. (A.7) reduces to

$$
\delta^{2}\left(A_{p(n, d)}^{\pi^{+}-\pi^{-}}\right)=\frac{4}{D^{2}} \frac{\left(N_{\uparrow \uparrow}^{\pi^{+}}-N_{\uparrow \uparrow}^{\pi^{-}}\right)^{2}\left[N_{\uparrow \downarrow}^{\pi^{+}}+N_{\uparrow \downarrow}^{\pi^{-}}\right]+\left(N_{\uparrow \downarrow}^{\pi^{+}}-N_{\uparrow \downarrow}^{\pi^{-}}\right)^{2}\left[N_{\uparrow \uparrow}^{\pi^{+}}+N_{\uparrow \uparrow}^{\pi^{-}}\right]}{\left[\left(N_{\uparrow \downarrow}^{\pi^{+}}+N_{\uparrow \uparrow}^{\pi^{+}}\right)-\left(N_{\uparrow \downarrow}^{\pi^{-}}+N_{\uparrow \uparrow}^{\pi^{-}}\right)\right]^{4}} .
$$

Let us now choose as the variables the set $X_{1}=N_{\uparrow \downarrow}^{\pi^{+}}-N_{\uparrow \downarrow}^{\pi^{-}}, X_{2}=N_{\uparrow \uparrow}^{\pi^{+}}-N_{\uparrow \uparrow}^{\pi^{-}}$, so that the equation (A.1) for asymmetry is rewritten as

$$
A_{p(n, d)}^{\pi^{+-} \pi^{-}}=\frac{1}{D} \frac{X_{1} L_{\uparrow \uparrow}-X_{2} L_{\uparrow \downarrow}}{X_{1} L_{\uparrow \uparrow}+X_{2} L_{\uparrow \downarrow}}
$$


Then Eq. A.5 gives:

$$
\begin{aligned}
& \delta^{2}\left(A_{p(n, d)}^{\pi^{+}-\pi^{-}}\right)= \\
& =\frac{4 L_{\uparrow \uparrow}^{2} L_{\uparrow \downarrow}^{2}}{D^{2}} \frac{X_{2}^{2} \delta^{2}\left(X_{1}\right)+X_{1}^{2} \delta^{2}\left(X_{2}\right)}{\left(X_{1} L_{\uparrow \uparrow}+X_{2} L_{\uparrow \downarrow}\right)^{4}},
\end{aligned}
$$

with

$$
\begin{aligned}
& \delta^{2}\left(X_{1}\right)=\delta^{2}\left(N_{\uparrow \downarrow}^{\pi^{+}}\right)+\delta^{2}\left(N_{\uparrow \downarrow}^{\pi^{-}}\right)-2 \operatorname{cov}\left(N_{\uparrow \downarrow}^{\pi^{+}}, N_{\uparrow \downarrow}^{\pi^{-}}\right), \\
& \delta^{2}\left(X_{2}\right)=\delta^{2}\left(N_{\uparrow \uparrow}^{\pi^{+}}\right)+\delta^{2}\left(N_{\uparrow \uparrow}^{\pi^{-}}\right)-2 \operatorname{cov}\left(N_{\uparrow \uparrow}^{\pi^{+}}, N_{\uparrow \uparrow}^{\pi^{-}}\right) .
\end{aligned}
$$

Again, with a standard assumption that the distributions of hadrons $N_{\uparrow \uparrow(\uparrow \downarrow)}^{\pi^{+}\left(\pi^{-}\right)}$are Poissonian:

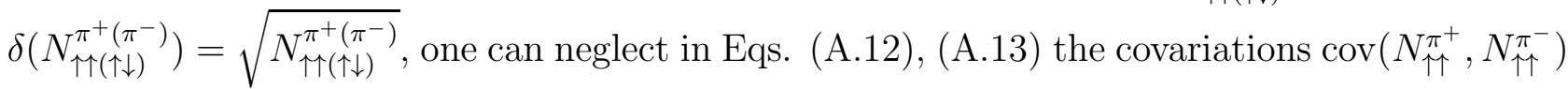
and $\operatorname{cov}\left(N_{\uparrow \downarrow}^{\pi^{+}}, N_{\uparrow \downarrow}^{\pi^{-}}\right)$. Then Eq. (A.11) exactly transforms to Eq. (A.7).

Let us involve an additional approximation (see [23, p.7)

$$
X_{1} \simeq X_{2} \simeq \tilde{Y} / 2
$$

where the quantity ${ }^{26} \tilde{Y}$ is defined as $\tilde{Y} \equiv\left(N_{\uparrow \downarrow}^{\pi^{+}}+N_{\uparrow \uparrow}^{\pi^{+}}\right)-\left(N_{\uparrow \downarrow}^{\pi^{-}}+N_{\uparrow \uparrow}^{\pi^{-}}\right) \equiv N^{\pi^{+}}-N^{\pi^{-}}$. Then Eq. (A.11) reads:

$$
\begin{aligned}
& \delta^{2}\left(A_{p(n, d)}^{\pi^{+}-\pi^{-}}\right)=\frac{1}{D^{2}} \frac{16 L_{\uparrow \downarrow}^{2} L_{\uparrow \uparrow}^{2}}{\tilde{Y}^{2}\left(L_{\uparrow \downarrow}+L_{\uparrow \uparrow}\right)^{4}} \delta^{2}(\tilde{Y}) \equiv \frac{16 L_{\uparrow \downarrow}^{2} L_{\uparrow \uparrow}^{2}}{D^{2}\left(L_{\uparrow \downarrow}+L_{\uparrow \uparrow}\right)^{4}} \frac{1}{\left(N^{\pi+}-N^{\pi-}\right)^{2}} \delta^{2}\left(N^{\pi+}-N^{\pi-}\right) \\
& =\frac{N^{\pi^{+}}+N^{\pi^{-}}}{\left(N^{\pi^{+}}-N^{\pi^{-}}\right)^{2}} \frac{16 L_{\uparrow \downarrow}^{2} L_{\uparrow \uparrow}^{2}}{D^{2}\left(L_{\uparrow \downarrow}+L_{\uparrow \uparrow}\right)^{4}} .
\end{aligned}
$$

With the SMC (COMPASS) target setup $L_{\uparrow \downarrow}=L_{\uparrow \uparrow}$ and

$$
\delta^{2}\left(A_{p(n, d)}^{\pi^{+}-\pi^{-}}\right)=\frac{1}{D^{2}} \frac{N^{\pi^{+}}+N^{\pi^{-}}}{\left(N^{\pi^{+}}-N^{\pi^{-}}\right)^{2}} .
$$

It is instructive to reproduce Eq. (A.16) choosing another variables in Eq. (A1): $\Delta N^{\pi^{+}}=$ $N_{\uparrow \downarrow}^{\pi^{+}}-N_{\uparrow \uparrow}^{\pi^{+}}, \Delta N^{\pi^{-}}=N_{\uparrow \downarrow}^{\pi^{-}}-N_{\uparrow \uparrow}^{\pi^{-}}, N^{\pi^{+}}=N_{\uparrow \downarrow}^{\pi^{+}}+N_{\uparrow \uparrow}^{\pi^{+}}, N^{\pi^{-}}=N_{\uparrow \downarrow}^{\pi^{-}}+N_{\uparrow \uparrow}^{\pi^{-}}$, so that with $L_{\uparrow \downarrow}=L_{\uparrow \uparrow}$ (COMPASS target setup) Eq. (A.1) for asymmetry reads

$$
A_{p(n, d)}^{\pi^{+}-\pi^{-}}=\frac{1}{D} \frac{\Delta N^{\pi^{+}}-\Delta N^{\pi^{-}}}{N^{\pi^{+}}-N^{\pi^{-}}} \equiv \frac{1}{D} \frac{\tilde{X}}{\tilde{Y}} .
$$

Then the respective statistical error look as

$$
\begin{aligned}
& \delta^{2}\left(A_{p(n, d)}^{\pi^{+}-\pi^{-}}\right)=\frac{1}{D^{2} \tilde{Y}^{2}}\left\{\delta^{2}\left(\Delta N^{\pi^{+}}\right)+\delta^{2}\left(\Delta N^{\pi^{-}}\right)+\frac{\tilde{X}^{2}}{\tilde{Y}^{2}}\left(\delta^{2}\left(N^{\pi^{+}}\right)+\delta^{2}\left(N^{\pi^{-}}\right)\right)\right\} \\
& \simeq \frac{1}{D^{2} \tilde{Y}^{2}}\left(1+\frac{\tilde{X}^{2}}{\tilde{Y}^{2}}\right)\left(N^{\pi^{+}}+N^{\pi^{-}}\right)
\end{aligned}
$$

\footnotetext{
${ }^{26}$ It is easy to see that $\tilde{Y}$ up to luminosities coincides with denominator $Y$ in the square brackets in Eq. A.1.
} 
where it is again adopted that distributions of hadrons $N_{\uparrow \uparrow(\uparrow \downarrow)}^{\pi^{+}\left(\pi^{-}\right)}$are Poissonian, so that $\delta\left(\Delta N^{\pi^{+}}\right)=$ $\delta\left(N^{\pi^{+}}\right)=\sqrt{N_{\uparrow \downarrow}^{\pi^{+}}+N_{\uparrow \uparrow}^{\pi^{+}}}, \delta\left(\Delta N^{\pi^{-}}\right)=\delta\left(N^{\pi^{-}}\right)=\sqrt{N_{\uparrow \downarrow}^{\pi^{-}}+N_{\uparrow \uparrow}^{\pi^{-}}}$and one can neglect $\operatorname{cov}\left(\Delta N^{\pi^{+}}, N^{\pi^{-}}\right)$ and $\operatorname{cov}\left(\Delta N^{\pi^{-}}, N^{\pi^{+}}\right)$. By virtue of Eq. (A.14),

$$
\left|\frac{\tilde{X}}{\tilde{Y}}\right|=\left|\frac{X_{1}-X_{2}}{X_{1}+X_{2}}\right|<<1,
$$

so that one can neglect ${ }^{27}(\tilde{X} / \tilde{Y})^{2}$ in Eq. (A.19). Thus, one again arrives at the approximate formula (A.16) for the error on the difference asymmetries.

\section{References}

[1] NMC collaboration (M. Arneodo et al.), Phys. Lett. B 364 (1995) 107;

FNAL E866/NuSea Collaboration (E.A. Hawker et al.), Phys. Rev. Lett. 80, (1998) 3715; HERMES collaboration (K. Ackerstaff et al.) Phys. Rev. Lett. 81, (1998) 5519.

[2] A.N. Sissakian, O.Yu. Shevchenko and O.N. Ivanov, Phys. Rev. D 68 (2003) 031502(R).

[3] HERMES collaboration (K. Ackerstaff et al.), Phys. Lett. B 464 (1999) 123.

[4] E. Christova and E. Leader, Nucl. Phys B 607 (2001) 369.

[5] SMC collaboration ( D. Adams et al.), Phys. Rev. D 56 (1997) 5330;

SMC collaboration (B. Adeva et al.), Phys. Lett. B 369 (1996) 93.

[6] SMC collaboration (B. Adeva et al.) Phys. Lett. B 420 (1998) 180.

[7] COMPASS collaboration (G. Baum et al.), "COMPASS: A proposal for a common muon and proton apparatus for structure and spectroscopy", CERN-SPSLC-96-14 (1996).

[8] D. de Florian, L.N. Epele, H. Fanchiotti, C.A. Garcia Canal, S. Joffily, R. Sassot, Phys. Lett. B 389 (1996) 358.

[9] D. de Florian, M. Stratmann and W. Vogelsang, Phys. Rev. D 57 (1998) 5811.

[10] D. de Florian and R. Sassot, Phys. Rev. D 62 (2000) 094025.

[11] J. Binnewies, B.A. Kniehl, G. Kramer, Z. Phys. C 65 (1995) 471.

[12] European Muon Collaboration ( M. Arneodo et al. ), Nucl. Phys. B 321 (1989) 541.

[13] L. Mankiewicz, A. Schafer, M. Veltri, Comput. Phys. Commun.71 (1992) 305-318.

[14] EMC collaboration (J, Ashman et al.), Z. Phys. C 52 (1991) 361 , CERN-PPE-91-53 (can be obtained electronically via KEK library page http://www-lib.kek.jp/KISS/kiss_prepri.html).

\footnotetext{
${ }^{27}$ Let us recall (see footnotes [13] and [15) that with the applied statistics $3 \cdot 10^{6}$ DIS events, the quantity $\tilde{Y} \equiv N^{\pi^{+}}-N^{\pi^{-}}$essentially differs from zero even in the vicinity of the minimal value $x_{B}=0.003$ accessible for measurement.
} 
[15] G. Ingelman, A. Edin, J. Rathsman Comput. Phys. Commun. 101 (1997) 108.

[16] M. Gluck, E. Reya, M. Stratmann, W. Vogelsang, Phys.Rev. D 63 (2001) 094005.

[17] M. C. Simani, Flavour decomposition of the nucleon spin at HERMES, Ph. D. thesis, Vrije Universiteit Amsterdam, 2002, (can be obtained electronically via "HERMES publications" internet page).

[18] S. Kretzer, Phys.Rev. D 62 (2000) 054001.

[19] M. Gluck, E. Reya, A. Vogt, Eur.Phys.J. C 5 (1998) 461.

[20] R.K. Ellis, M.A. Furman, H.E. Haber and I. Hinchliffe, Nucl. Phys. B 173 (1980) 397.

[21] E. Leader, A. Sidorov, D. Stamenov Phys.Rev. D 58 (1998) 114028.

[22] E. Leader, A. Sidorov, D. Stamenov Eur. Phys. J. C23 (2002) 479 (LSS2001);

Asymmetry Analysis Collaboration (Y. Goto et al), Phys. Rev. D 62 (2000) 034017 (AAC2000);

Asymmetry Analysis Collaboration (M. Hirai et al), Phys. Rev. D 69 (2004) 054021 (AAC2003).

[23] J.M. Le Goff and J. Pretz, COMPASS note 2004-4, can be obtained electronically via COMPASS collaboration site http://wwwcompass.cern.ch, direct link is http://wwwcompass.cern.ch/compass/notes/2004-4/2004-4.ps. 